\title{
光致变色双呋喃俘精酸酐的合成及其在平行 图象存储中的应用
}

问起强 ${ }^{(1)}$ 陈 懿 ${ }^{(1)}$ 姚保利 ${ }^{(2 * *}$ 雷 铭 $^{(2)}$ 王英利 ${ }^{(2)}$
黎甜楷

(1)中国科学院理化技术研究所, 北京 100101; (2)中国科学院西安光学精密机械研究所, 西安 710068)

\begin{abstract}
摘要通过两步 Stobbe 缩合反应合成了一种双杂环俘精酸酐化合物, E,E-3,4-二 [1-(2,5-二甲基-3-呋喃基)乙叉]-3,4-二氢呋喃-2,5-二酮(双呋喃俘精酸酐). 通过 X 射线单 晶衍射, 对目标产物的分子结构进行了研究, 结果表明在该化合物中, 2 个反应中心的 距离分别是 0.3394 和 $0.3406 \mathrm{~nm}$, 有利于光环合反应. 研究了该化合物在不同溶剂中的 光致变色性行为, 并对目标产物在平行图象光信息存储中的应用进行了探索.
\end{abstract}

\section{光键词双呋喃俘精酸酐 光致变色 平行图象 光信息存储 单晶结构}

近年来, 由于光致变色材料在光信息存储 ${ }^{[1 \sim 5]}$ 、光开关、分子识别 ${ }^{[6 \sim 8]}$ 等诸多领域具有潜在 的应用前景, 有机光致变色化合物受到了广泛的关注. 在这个领域, 一个重要的研究方向是杂 环俘精酸酤化合物的合成及其应用.

自从 Heller ${ }^{[9]}$ 在 20 世纪 70 年代末首先指出杂环俘精酸酐化合物有可能用于光信息存储以 来, 相继报道了大量关于杂环俘精酸酐的合成及其物化性质的研究文章 ${ }^{[1,2]}$, 也陆续开发出一 些稳定性好, 抗㽻劳好的俘精酸酐类化合物, 使有机光致变色材料在实用化的方面有了长足 的进步. 但就其内容来说, 所合成的化合物多限于单杂环取代的俘精酸酐类化合物, 有关双杂 环俘精酸酐化合物的报道很少.

与单杂环取代俘精酸酐相比, 双杂环俘精酸酐分子内每一个甲叉碳上都有一个取代的杂 环, 从理论上讲, 可能组成 2 个可以进行电环化反应的体系(类似全顺式己三烯结构), 更有利 于光致变色反应的进行. 然而, 这类分子结构具有更大的空间位阻, 带来了合成上的难度. 迄 今为止, 有关这方面的研究报道很少.

1999 年, Kiji 等人 ${ }^{[10]}$ 报道了通过 1,4-双杂环取代的丁炔-1,4-二醇的羰基化的方法来合成双 杂环俘精酸酐化合物, 反应在钯 $(\mathrm{Pd})$ 催化下, 在高温高压下进行. 此方法为双杂环俘精酸酐的 合成开辟了一条新的路径, 但合成条件苛刻, 较难推广.

本文首次报道使用传统的 Stobbe 缩合反应的方法, 通过两步 Stobbe 缩合反应合成双呋喃 俘精酸酐化合物. 所得结果与 Kiji 报道的结果的不同之处在于: Kiji 方法所得的双杂环俘精酸 酤化合物的结构为 $Z Z$ 式, 而本实验合成的双呋喃俘精酸酐化合物的结构为 $E E$ 式. 此外, 本文

2003-01-20 收稿, 2003-04-23 收修改稿

* 国家重点基础研究 “九七三”计划(G 1999033005)和国家自然科学重点基金(批准号: 29832030)资助项目

** 联系人, E-mail: mgf206@ipc.ac.cn 
还将报道目标产物的光致变色性质及其在平行图象光信息存储中的应用探索结果.

\section{1 实验}

\section{1 试剂}

甲苯, 乙醇, 四氢呋喃均为分析纯, 北京化工厂, 使用前经脱水处理. 其他试剂均为市购, 使用前未经过进一步处理.

\section{2 实验仪器}

${ }^{1}$ H NMR 由 Varian Germina-300 光谱仪测定; MS 由 Trio-2000 气质连用仪测定; 所有紫外 光谱均由 Hitachi U-3010 紫外可见光度计测定; 光反应在室温下进行, $30 \mathrm{~W}$ 的低压求灯作为 光源. 目标产物的单晶结构由 Enraf-Nonius CAD4 四圆衍射仪测定. 用于平行存储的实验薄片 按以下步骤制备: 目标化合物 $3 \mathrm{mg}$ 溶解于 $0.1 \mathrm{~mL}$ 聚甲基丙稀酸甲酯的环己酮(10\%质量浓度) 溶液中, 均匀地涂布在 $25 \mathrm{~mm} \times 25 \mathrm{~mm} \times 1.5 \mathrm{~mm}$ 大小的光学玻璃片上, 在室温下避光干燥、保 存. 光致变色高分子膜的厚度大概是 $30 \mu \mathrm{m}$.

平行存储记录实验装置见图 1, 激光波长为 $514 \mathrm{~nm}$, 平均功率为 $200 \mathrm{~mW}$, 读出用 CCD 摄 象机. 信息图象由计算机 I 控制, 实验薄片被固定在两维微调系统上, 其在 $Y-Z$ 的方向可以由 计算机 II 来调节. 记录能量 $93 \mathrm{~mJ}$, 读出能量 $0.3 \mathrm{~mJ}$.

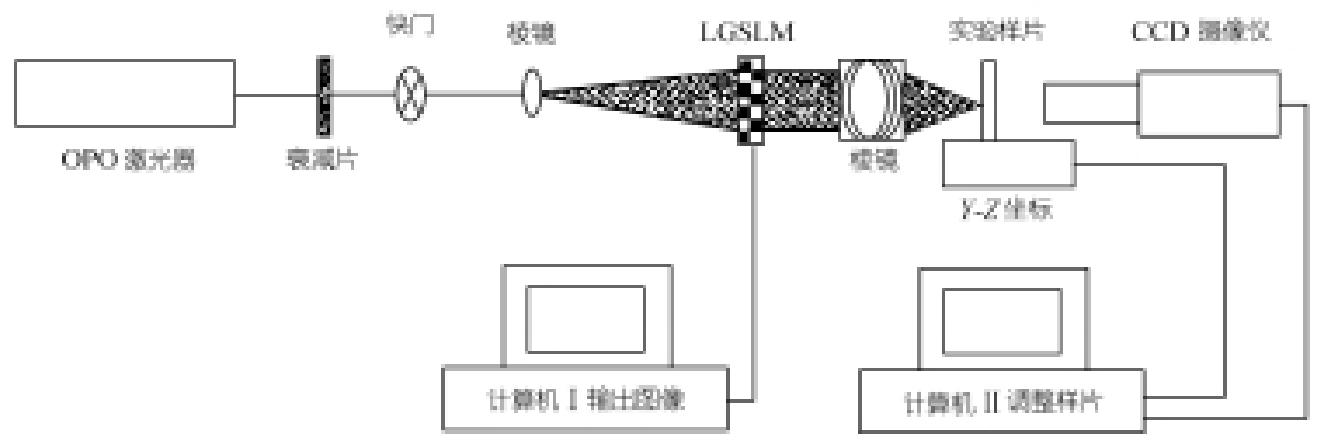

图 1 平行存储图象记录系统

\section{$1.3 E, E$ 双 2,5-二甲基呋喃取代俘精酸酐的合成}

\subsubsection{2-[-1-(2,5-二甲基-3-呋喃)乙叉]丁二酸二乙酯的合成 金属钾 $4 \mathrm{~g}$ 溶解在 $100 \mathrm{~mL}$ 无水} 叔丁醇中. 2,5-二甲基-3-乙酰基呋喃 $9.2 \mathrm{~mL}(0.075 \mathrm{~mol}$ )和丁二酸二乙酯 $14 \mathrm{~mL}(0.082 \mathrm{~mol})$ 和 15 $\mathrm{mL}$ 叔丁醇混合后, 滴加于上述叔丁醇钾溶液中, 保持溶液缓慢回流. 加完后继续回流 $4 \mathrm{~h}$, 然 后冷却到室温. 用浓度为 $5 \mathrm{~mol} / \mathrm{L}$ 的盐酸酸化到 $\mathrm{pH}$ 值为 $5 \sim 6$, 减压蒸去溶剂. 将残余物溶解于 $50 \mathrm{~mL}$ 乙醚, 用饱和碳酸钠溶液 $(10 \%)$ 萃取 3 次 $(60 \mathrm{~mL} \times 3)$, 合并水相, 用 $5 \mathrm{~mol} / \mathrm{L}$ 盐酸酸化至 强酸性 $(\mathrm{pH}<1)$, 上层出现红棕色油状物, 分出有机层. 水相用乙醚萃取 3 次 $(30 \mathrm{~mL} \times 3)$, 合并 有机相, 用无水硫酸钠干燥后, 减压蒸去溶剂, 得到 2-[-1-(2,5-二甲基呋喃-3-基)乙叉 $]$ 取代的 丁二酸单乙酯.

将所得单乙酯加人 $60 \mathrm{~mL}$ 无水乙醇中, 加人 $3 \mathrm{~mL}(98 \%)$ 浓硫酸, 加热回流 $6 \mathrm{~h}$, 减压除去 过量的乙醇后, 冷至室温. 倾人到 $50 \mathrm{~mL}$ 冰水中, 分出有机相(下层). 水层用乙醚萃取 3 次(30 
$\mathrm{mL} \times 3)$, 与有机相合并. 分别用 $60 \mathrm{~mL}$ 饱和的碳酸氢钠水溶液和水洗涤有机相至中性, 干燥后 蒸除溶剂, 得到黄色油状液体. 油状物用硅胶柱层析分离, 淋洗液为乙酸乙酯和石油醚的混合 物 $(\mathrm{v} / \mathrm{v}=1 / 4)$, 得 2-[-1-(2,5-二甲基 3-呋喃)乙叉]丁二酸二乙酯(收率 54\%).

${ }^{1} \mathrm{H} \operatorname{NMR}\left(\delta, \mathrm{CDCl}_{3}\right): 1.245\left(2 \mathrm{t}, 6 \mathrm{H}, \mathrm{CH}_{3}\left(\mathrm{CH}_{2} \mathrm{O}\right)\right) ; 2.193$ (s, 3H, MeC=); 2.282 (s, 3H, furan-5$\mathrm{Me}) ; 2.376\left(\mathrm{~s}, 3 \mathrm{H}\right.$, furan-2-Me); 3.183(s, 2H, $-\mathrm{CH}_{2}-$ ); $4.063 \sim 4.244$ (2q, 4H, $\left.-\mathrm{CH}_{2} \mathrm{O}-\right)$; 6.353 (s, 1H, furan-4-H).

1.3.2 $E, E-3,4-$ 双-二[1-(2,5-二甲基-3-呋喃基)乙叉]-3,4-二氢呋喃-2,5-二酮的合成 在一干 燥的三颈烧瓶中于氮气保护下加人氢化钠 $2 \mathrm{~g}(80 \%)$ 和无水甲苯 $10 \mathrm{~mL}$, 将 2-[-1-(2,5-二甲基 3呋喃基)乙叉]丁二酸二乙酯 $4 \mathrm{~g}$ 和 2,5-二甲基-3-乙酰基呋喃 $1.9 \mathrm{~g}$ 的 $40 \mathrm{~mL}$ 无水甲苯溶液滴加 到三颈烧瓶中的氢化钠悬浮液中, 同时加 1 滴无水乙醇引发反应. 滴加完毕后, 于 $30 \sim 35^{\circ} \mathrm{C}$ 下 搅拌 $5 \mathrm{~d}$. 将反应混合物倒人 $50 \mathrm{~mL}$ 冰水中, 分出水相, 有机相用 $30 \mathrm{~mL}$ 的饱和碳酸钠水溶液 萃取 2 次, 分离有机相, 水相合并, 用 $5 \mathrm{~mol} / \mathrm{L}$ 的盐酸酸化至强酸性 $(\mathrm{pH}<1)$. 分出反应所得油 状 3,4-双-[1-(2,5-二甲基-3-呋喃基)乙叉]丁二酸单乙酯, 水相用 $50 \mathrm{~mL}$ 的乙酸乙酯萃取 3 次, 合 并有机相并用无水硫酸镁干燥. 滤出干燥剂, 蒸除溶剂, 所得胶状半酯在 $5 \mathrm{~g}$ 氢氧化钾的 50 $\mathrm{mL}$ 无水乙醇中回流 $5 \mathrm{~h}$. 冷却, 减压蒸去大部分溶剂, 残余物倒人水中, 用 $5 \mathrm{~mol} / \mathrm{L}$ 的盐酸酸 化至强酸性 $(\mathrm{pH}<1)$, 获得 2,3-双 [(2,5-二甲基-3-呋喃基)乙叉 $] 丁 二$ 酸. 干燥的二酸溶解在 $40 \mathrm{~mL}$ 无水四氢呋喃中, 加人 $1.0 \mathrm{~g}$ 的二环己基碳二亚胺, 搅拌 $12 \mathrm{~h}$. 蒸除溶剂, 残余物经硅胶柱分离, 淋洗液为乙酸乙酯和石油醚 $\left(60 \sim 90^{\circ} \mathrm{C}\right)$ 的混合物 $(\mathrm{v} / \mathrm{v}=1 / 4)$, 粗产物在氯仿和石油醚混合溶剂中 重结晶, 得到纯净标题化合物, 淡黄色晶体. 熔点: $163 \sim 165^{\circ} \mathrm{C}$, 产率 $6.4 \%$. ${ }^{1} \mathrm{H} \mathrm{NMR}\left(\delta, \mathrm{CDCl}_{3}\right)$ : 1.883(s, 6H, Me=); 2.127(s, 6H, furan-2-Me); 2.523(s, 6H, furan-5- Me); 5.494(s, 2H, furan-4-H). $\operatorname{MS}(m / z): 340\left(\mathrm{M}^{+}\right), 325\left(\mathrm{M}^{+}-15\right)$.

\section{2 结果与讨论}

\section{1 目标化合物的合成路线}

如图 2 所示, 我们使用 3-乙酰基-2,5-二甲基呋喃作为起始原料, 与丁二酸二乙酯发生两步 的 Stobbe 缩合反应得到目标化合物. 其中在第一步反应中使用叔丁醇钾作为有机碱, 所得半 酯在乙醇中酯化, 得到所需要的中间体 2-[-1-(2,5-二甲基-3-呋喃基)乙叉]丁二酸二乙酯. 此中 间体再与另一分子的 3-乙酰基-2,5-二甲基呋喃在氢化钠催化下, 在甲苯中缩合. 反应产物经

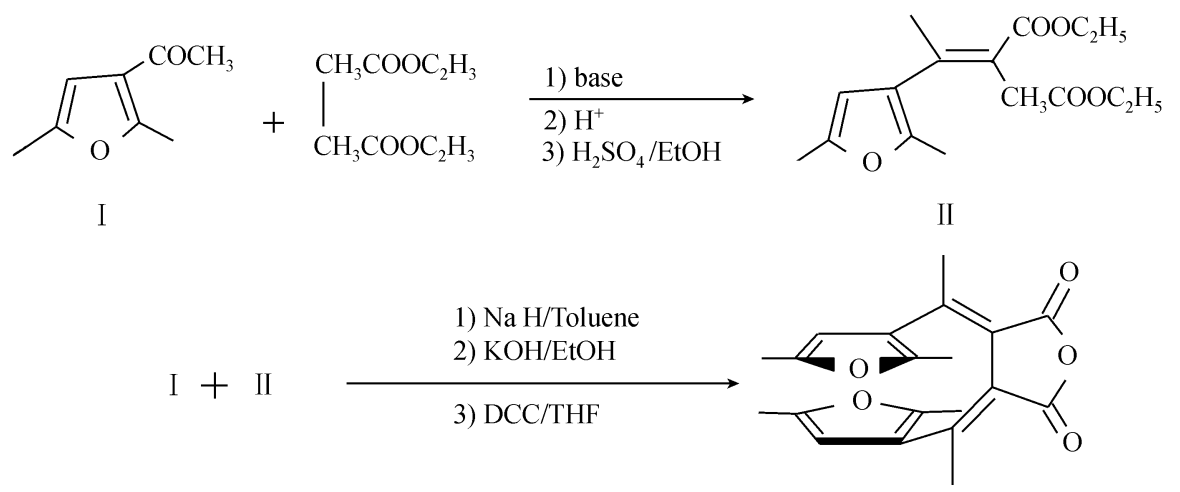

图 2 目标化合物的合成路线 
㿝化得到 2,3-双-二-[(2, 5-二甲基-3-呋喃基)乙叉]丁二酸, 再经脱水反应, 得到最终目标化合 物.

\section{2 目标化合物的单晶结构}

Kiji 等人 ${ }^{[10]}$ 报道了通过 2,3-双杂环取代的丁炔-1,4-二醇的羰基化反应来合成双呋喃俘精 酸酐化合物, 产物经重结晶后, 得到 $Z Z$ 异构体作为惟一的产物. Yokoyama 等 ${ }^{[11]}$ 报道使用同样 的方法制备双噻吩化合物时, 得到的主要产物是 $E E$ 式异构体. 通常来说, 使用 Stobbe 缩合反

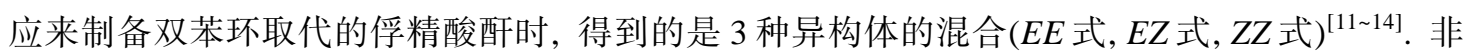
常有趣的是, 在我们的实验中, 只有 $E E$ 式异构体被获得. 我们通过 $\mathrm{X}$ 射线晶体衍射方法研究 目标化合物的分子结构. 进一步证实了用核磁共振方法可得出的分子结构结果. 单晶数据如 下: $\mathrm{C}_{20} \mathrm{H}_{20} \mathrm{O}_{5}$, 分子量= 340.46, 三斜晶系, 空间群 $\mathrm{P}-1, a=1.1009(1), b=1.1404(2), c=$ $0.7601(1) \mathrm{nm}, \alpha=102.599(5)^{\mathrm{o}}, \beta=106.909(11)^{\mathrm{o}}, \gamma=87.388(6)^{\mathrm{o}}, Z=2, V=0.8909(3) \mathrm{nm}^{3}, T=$ $293 \mathrm{~K}, D_{\mathrm{e}}(\mathrm{cal})=1.269 \mathrm{mg} / \mathrm{mm}^{3}, u=0.091 \mathrm{~mm}^{-1}$, 总反射点 5865 , 单反射点 $3611\left(R_{\mathrm{int}}=0.0342\right)$, $R / R_{\mathrm{w}}[I>2 \delta(I)]=0.0512 / 0.1217$, 数据收集范围 $2.6^{\circ}<\theta<27^{\circ}$, 参数范围 $-14 \leqslant h \leqslant 14,-14 \leqslant k \leqslant 14$, $-9 \leqslant l \leqslant 9$, 依据经验校正吸收. 晶体结构及键长键角数据见图 3 及表 1 .

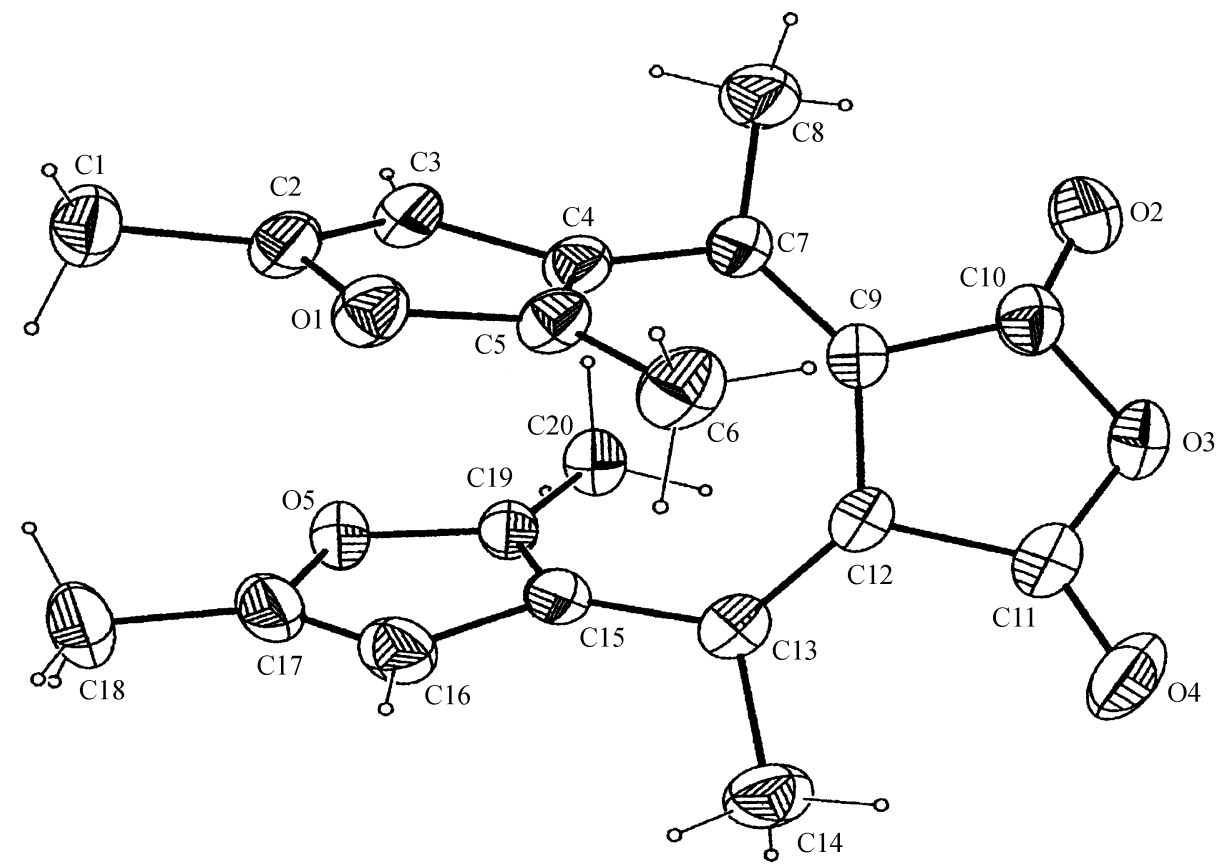

图 3 双呋喃化合物的单晶结构

仔细研究化合物的单晶结构, 我们发现 2 个呋喃环几乎是平行的 (二面角是 $13.6^{\circ}$ ), $\pi-\pi$ 轨 道在空间上有一定程度的重叠 (见图 4). 可能正是由于这种 $\pi-\pi$ 轨道之间的互相作用, 在通过 Stobbe 缩合反应制备双杂环俘精酸酐的反应过程中稳定了 $E E$ 构型, 使得 $E E$ 构型比其他构型 更稳定，因此作为惟一的产物被分离获得. 


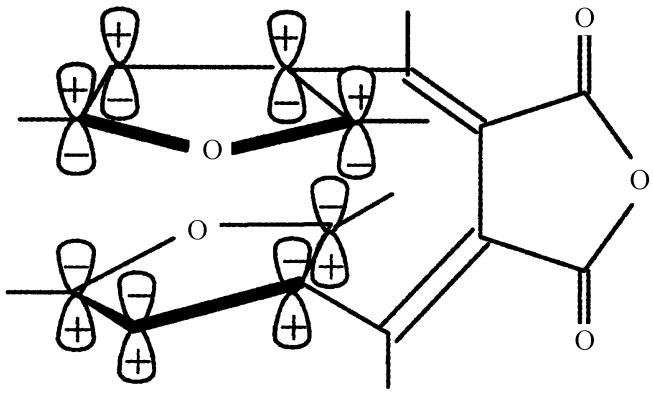

图 42 个呋喃环 $\pi-\pi$ 轨道之间的重叠
表 1 目标化合物的部分键长和键角数据

\begin{tabular}{llll}
\hline \multicolumn{2}{c}{ 键长 $/ \mathrm{nm}$} & \multicolumn{2}{c}{ 键角 $\left./{ }^{\circ}\right)$} \\
\hline C15-C13 & $0.1455(3)$ & C7-C9-C12 & $13.293(19)$ \\
C13-C12 & $0.1364(3)$ & C13-C12-C9 & $13.220(18)$ \\
C12-C9 & $0.1478(3)$ & C15-C13-C12 & $12.362(18)$ \\
C9-C7 & $0.1394(3)$ & C4-C7-C9 & $12.288(19)$ \\
C7-C4 & $0.1454(3)$ & C14-C13-C12 & $12.24(2)$ \\
C15-C19 & $0.1357(3)$ & C8-C7-C9 & $12.22(2)$ \\
C4-C5 & $0.1365(3)$ & & \\
\hline
\end{tabular}

从表 1 的数据我们可以看出 C7-C9-C12 和 C13-C12-C9 的键角变大 $\left(132.93^{\circ}\right.$ 和 $\left.132.20^{\circ}\right)$, 这 是由于 2 个呋喃环的空间位阻的影响. 在 C5-C4-C7-C9-C12-C13 和 C19-C15-C13-C12-C9-C7 2 个六碳体系中存在着单键和双键的交替, 根据 Woodward-Hoffmann $4 n+2$ 电环化规则 ${ }^{[15]}$, 光 环合反应发生在 C19 和 C7 原子(或者 C5 和 C13 原子)间, 它们之间的距离是 $0.3393 \mathrm{~nm}$ (或者 $0.3406 \mathrm{~nm})$, 适合于光环合反应的进行.

\section{3 光致变色性质}

在不同的溶剂中，目标化合物均表现了良好的光致变色性. 在甲苯溶液中的结果，如图 5 所示.

目标产物 (open-form)的最大吸收峰 $\left(\lambda_{\max }\right.$ ) 在 $368 \mathrm{~nm}$, 成色体(closed-form)的 $\lambda_{\max }$ 在 $489 \mathrm{~nm}$. 用紫外光激发(成色过程)时, 可以检测到紫外区的吸收逐渐降低, 而可见区出现的吸收峰强度 逐渐增加, 直至光稳态, 呈现从淡黄色到红色的变化. 当用可见光漂白有色体时(消色过程)则 可以检测到相反的光谱变化. 在 $411 \mathrm{~nm}$ 处存在明显的等光密度点, 这说明在我们的实验条件 下仅观察到成色体和开环体之间彼此转化，预期该化合物的抗㽻劳性能很好.

表 2 目标化合物(开环体)及其成色体(闭环体)的 $\lambda_{\text {max }}$ 在不同极性溶剂中的变化

\begin{tabular}{ccc}
\hline \multirow{2}{*}{ 溶剂 } & \multicolumn{2}{c}{$\lambda_{\max } / \mathrm{nm}$} \\
\cline { 2 - 3 } & 开环体 & 闭环体 \\
\hline 乙醇 & 370 & 512 \\
乙氧 & 368 & 512 \\
二氧六环 & 364 & 498 \\
甲苯 & 368 & 489 \\
环己烷 & 360 & 480 \\
\hline
\end{tabular}

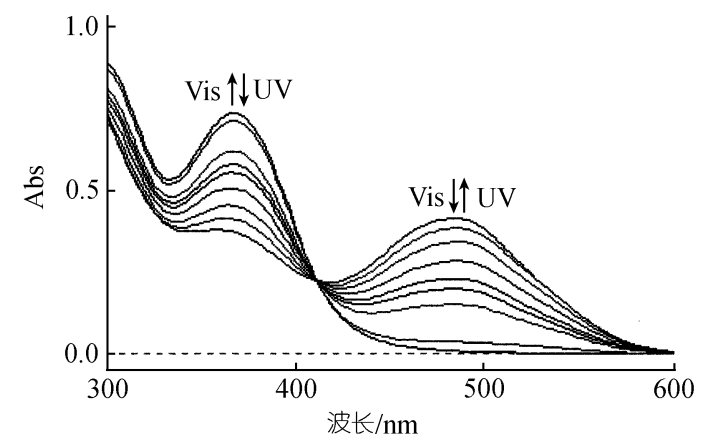

图 5 目标化合物的甲苯溶液中在 $254 \mathrm{~nm}$ 紫外光 和可见光照射下吸收光谱的变化(浓度为 $1.12 \times$ $10^{-4} \mathrm{~mol} / \mathrm{L}$ )

表 2 给出了在不同溶剂中的吸收光谱数据数据, 我们可以看到双呋喃俘精酸酐化合物 (open-form)和它的成色体(closed- form)的 $\lambda_{\text {max }}$ 随溶剂极性的变化而产生的变化. 结果指出溶剂 极性的变化对闭环体的影响比对开环体的影响更大, 这说明闭环体与开环体相比具有更好的 
平面性, 更易被极化, 所以对溶液的极性变化 更为敏感.

\section{4 在平行光信息存储中的应用}

自从 Heller 等人 ${ }^{[9]}$ 第一次提出具有热不可 逆性的俘精酸酤类化合物可能作为光信息存储 材料以来, 在这方面已经进行了大量的研究 ${ }^{[1-5]}$. 作为记录介质一般必须满足以下 4 点要求: 一 是热不可逆性, 二是高的图像分辨率, 三是开 环体和闭环体有明显的颜色变化, 四是高的光 反应量子产率.

本文仅就使用双呋喃俘精酸䣶化合物作为 平行光信息存储材料的初步实验结果进行报道,

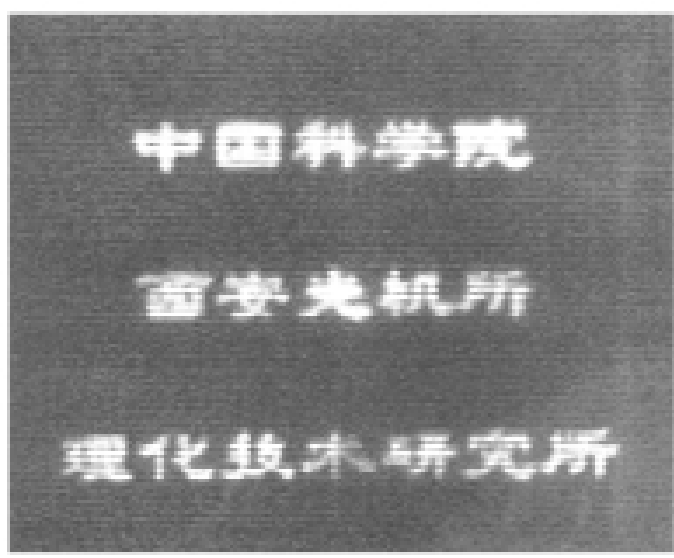

图 6 平行存储图像(图像大小 $460 \mu \mathrm{m} \times 350 \mu \mathrm{m})$ 在目标产物的聚甲基丙稀酸甲酯薄膜中被记录信息的原理性图像见图 6 , 更加深人的研究工 作还在继续当中.

\section{3 结论}

我们通过分步 Stobbe 缩合反应合成了 1 个分子内具有高空间位阻的化合物一一双呋喃取 代俘精酸酐. 研究了目标产物的分子结构及其在不同溶剂中的光致变色性能, 结果指出, 该化 合物可能具有很好的抗瘦劳性能. $\mathrm{X}$ 射线单晶分析结果说明, 目标产物具有 $E, E$-式结构, 有利 于光致变色反应的发生. 对目标产物用作平行存储介质进行了探索性的研究第一次实现了平 行图象存储.

\section{参考 文 献}

1 Fan M G, Yu L H, Zhao W L. Fulgide Family Compounds: Synthesis, Photochromism, and Application. Organic Photochromic and Thermochromic Compounds. Part IV. New York: Plenum Press, 1999. $141 \sim 186$

2 Yokoyama Y. Fulgides for memories and switches. Chem Rev, 2000, 100: 1717 1739

3 Feringa B L, Delden R A, Koumura N, et al. Chiroptical molecular switches. Chem Rev, 2000, 100: $1789 \sim 1816$

4 Irie M. Diaryethene for memories and swithes. Chem Rev, 2000, 100: 1685 1716

5 Kawata S, Kawata Y. Three dimensional optical data storage using photochemical materials. Chem Rev, 2000, 100: 1777 1788

6 Cabrera I, Dittrich A, Ringsdorf H. Thermally irreversible photochromic liquid crystal polymers. Angew Chem Int Ed Engl, 1991, 30: $76 \sim 78$

7 Willner I, Rubin S, Wonner J, et al. Photoswitchable binding of substrates to proteins: Photoregulated binding of a-D-mannopyranose to concanvalin a modified by a thiophenefulgide dye. J Am Chem Soc, 1992, 114: $3150 \sim 3151$

8 Huck N P M, Jager W F, De Lange B, et al. Dynamic control and amplification of molecular chirality by circular polarized light. Science, 1996, 273: $1686 \sim 1688$

9 Heller H G. The development of photochromic compounds for use in optical information storage. Chemistry and Industry, 1978, 18: $193 \sim 196$

10 Kiji J, Okano K, Takemoto A. A convenient and general synthetic method for photochromic fulgides by palladium-catalyzed carbonylation of 2-butyne-1,4-diols. Mol Cryst Liq Cryst, 2000, $344: 235 \sim 240$ 
11 Yokoyama Y, Sagisaka T, Yamaguchi Y, et al. Highly diastereoselective photochromic cyclization of a bisthienylfulgide. Chem Lett, 2000, $220 \sim 221$

12 Ilge H D. Photochemistry of phenyl fulgides. XX. Absence of activation barriers for photoisomerization reactions in the excited single state of extremely hindered $\alpha, \delta$-di-(4-alkoxyphenyl) fulgide isomers. J Photochem, 1986, 33: 333 347

13 Ilge H D, Kaschke M, Khechinashvili D. Photochemistry of phenyl fulgides. XXI: Ultrafast photoisomerization and radiationless deactivation processes in $\alpha, \delta$-di-(4-al-koxyphenyl) fulgides and $\alpha, \delta$-difluorenyl fulgide. J Photochem, 1986, 33 : $349 \sim 358$

14 Heller H G, Szewczyk M. Synthesis, stereochemistry and isomerisation of $\alpha$-phenyl-ethylidene-succinic esters and related compounds. J Chem Soc, Perkin Trans I, 1974, $1483 \sim 1487$

15 Whittall J. Photochromism: Molecules and System. Amsterdam: Elsevier, 1990. $467 \sim 492$ 